Article

\title{
Applying Semantic Network Analysis to Develop User Experience Assessment Model for Smart TV
}

\author{
Ji Hyoun Lim ${ }^{1}$, Ye Lim Rhie ${ }^{2}$ and Jaehyun Park ${ }^{3, *(1)}$ \\ 1 Human Factors Engineer in San Francisco Bay Area, San Francisco, CA 94080, USA \\ 2 Department of Industrial Engineering, Seoul National University, Seoul 08826, Korea \\ 3 Department of Industrial \& Management Engineering, Incheon National University, Incheon 22012, Korea \\ * Correspondence: jaehpark@inu.ac.kr; Tel.: +82-32-835-8867
}

Received: 13 July 2019; Accepted: 7 August 2019; Published: 12 August 2019

\begin{abstract}
The user experience (UX) concept has widely been adopted in the field of development and evaluation of products and services. One reason behind this emerging trend is the limitation in traditional usability evaluation methods (UEMs), which emphasizes performance measures such as task completion time and error rate. To overcome the limitation, users' perceived value must be set to reflect users' various experiences. In order to address this issue, this study proposes a network analysis-based method to investigate a value structure to assess the level of user experience in using a product. Gesture interaction on a smart TV is used as a case study to demonstrate the process of obtaining a quantified value structure from the transcriptions of users' in-depth interview. A major contribution of this study lies in the transformation from qualitative data to a quantified value structure. Product or service designers, in the industrial field, can develop value structure based on network analysis when evaluating their design alternatives within a properly fitted evaluation framework.
\end{abstract}

Keywords: user experience; user value; semantic network analysis; smart TV; gesture interaction

\section{Introduction}

The concept of user experience (UX) was introduced to overcome the shortcomings of traditional usability evaluation methods (UEMs). [1] defines usability as "the extent to which a product can be used by specified users to achieve specified goals with effectiveness, efficiency, and satisfaction in a specified context of use." Although some researchers have tried to embrace constructs such as impression and image to explain the concept of usability [2,3], in general, usability is considered as a performance-related measure which hardly represents the various contexts of use that exist for products or services.

The definitions of UX are diverse and abstract. [4] defines UX as "a person's perceptions and responses that result from the use or anticipated use of a product, system or service." The definition could be more abstract as "an overarching experience that consists of all aspects of users' interaction with a product or service" [5-9].

However, because of its ambiguous and abstract aspects, quantifying the level of UX is difficult. There is no general consensus among researchers on the specific elements which compose UX. For instance, [8] decomposed UX into elements consisting of usability, affect, and user value, while [10] suggested that three perspectives compose UX: beyond the instrumental, emotion and affect, and the experiential. Furthermore, researchers have reported that UX depends on temporality and varies over time $[11,12]$.

Consequently, in order to evaluate a level of UX in the use of product or service, researchers must first identify the UX elements and then decide how to quantify the elements. Even if the specific elements into which UX should be decomposed are unclear, value as a general term is undoubtedly 
related to UX. Value, if it can be comprehensively extracted from users and can be measured, is an important measure that represents users' perceptions and responses toward the product or service.

In the fields of marketing, psychology, and industrial design, many studies have focused on defining and quantifying the value. [13] suggested eighteen terminal values (e.g., true friendship, mature love, and self-respect) and eighteen instrumental values (e.g., cheerfulness, ambition, and forgiveness) that refer to desirable end-states of existence and modes of behavior, responsively. Although these values were widely adopted by various value researchers, many studies focused on the individual aspect rather than the product-related aspect. [14] associated these values with product choice. Note that $[15,16]$ defined user value as "desirable states of existence or modes of behavior which are satisfied when using a certain product or service." Recently, values have come to be recognized as important measures to explain users' product or service experiences.

The objective of this study is to suggest a practical approach to developing the value structure for a specific product or service usage. To formulate the value structure, this study proposes to apply co-occurrence-based network analysis on users' descriptions about their experiences of interaction with specific product and service in a given context. Since the proposed method adopts the network analysis, a series of mathematical analysis could be used in the analysis of keywords, without predetermining a set of keywords of interest. To demonstrate the proposed process of developing the value structure, gesture interaction adopted in smart TV was used as a case study. The various advantages and limitations of a value structure that contains mathematical information are also discussed.

\section{Network Analysis for UX Assessment}

Evaluating product or service experiences is difficult because of fundamental differences among individuals. People have different backgrounds and gain different experiences in various contexts, and tradition, common sense, and ideology have direct or indirect control over actions and thoughts with respect to all actions (e.g., purchase of products or services) [17]. Determining which values are important and how important they are is the key to quantifying the UX of products or services.

Although there are various methods for evaluating UX, there is no single method that is suitable for diverse products or services. Usually, well-defined hierarchical structures delivered by professionals have been used in the field of human-computer interaction, for instance, performance and image/impression dimensions [2]; ten usability heuristics [18]; effectiveness, efficiency, and satisfaction [1]; and usability, affect, and user value [8]. For the products or services which had not been considered in the development of the hierarchical structures, these measures do not capture some aspect of UX. For example, to some people, performance dimensions are not important when they choose clothes, while the performance dimensions such as reaction times or errors $[19,20]$ are still important across medical devices.

This study investigates the ability of network analysis to build a value structure, as an equipment of quantifying user value. The structure is composed of values that can be used to evaluate the UX of a product or service and may vary over contexts. Thus, a UX assessment method should efficiently and effectively capture unsettled evaluation dimensions such as the value structure, in contrast to traditional evaluation methods that settle evaluation dimensions, such as Nielsen's heuristics (e.g., visibility of system status, consistency and standards, and error prevention) [18]. Network analysis can be a potential alternative to these methods.

Network analysis has been widely employed to determine the relationships between social entities such as humans and other social animals. Since [21] first adopted a network approach, many researchers in the field of sociology have used so-called social network analysis [22-25]. Network analysis has been adopted in other areas including molecular network and communication [26,27]. In these cases, nodes in network structures can represent various targets: one study defined nodes as biomolecular species, and the other defined nodes as web pages. This adaptability allows network analysis to be applied to various fields. 
The most relevant network studies to UX assessment is semantic network analysis. With prominent propositions that memory structure connects individual items [28] and activation of a node in the network spreads to another node [29], efforts on semantic network analysis have been carried out in the field of psychology, information science, and computer science. Recently, studies about semantic network analyses focused on comparing products, services, or brandings [30,31] and improving the reliability of an application [32,33]. However, few studies have existed to apply semantic network analysis to UX assessment.

\section{Empirical Model with Value Structure}

In this study, a value structure is a set of hierarchical measures that are suitable for a certain product or service at that time. One of the most important reasons that the structure consists of values is that a product or service will eventually be purchased based on a user's values [14]. Values have been out of favor as usability or UX measures because of their low quantifiability. If this problem can be solved, values deserve to be included as UX measures.

A value structure consists of core values and associated values (Figure 1). Core values can be defined as the most influential values of a certain product or service, while associated values are values that have a relationship with the core values. By definition, each associated value may be related to more than two core values. Overall, a UX is assumed to be composed of core values. Of course, a UX may vary over time and individuals.

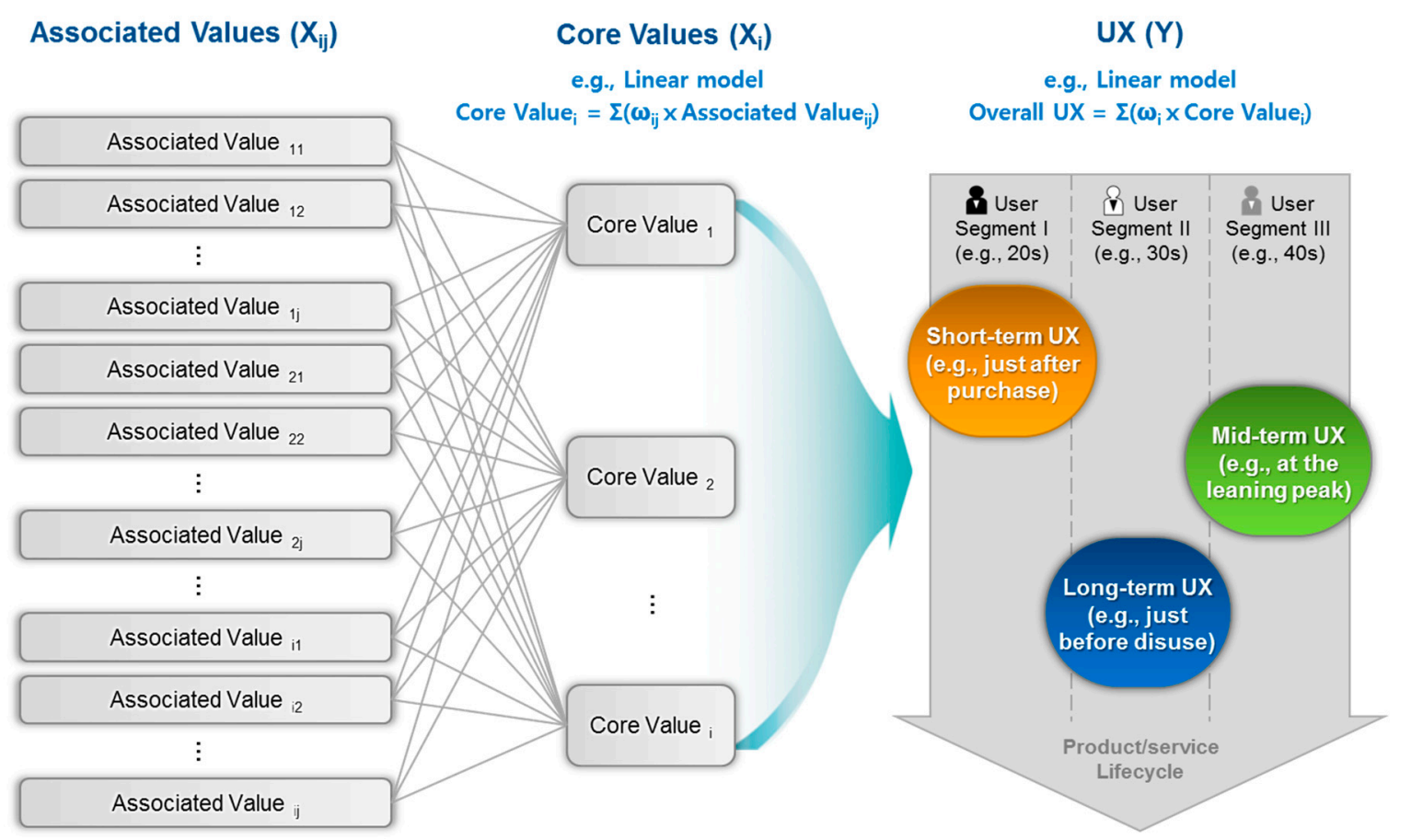

Figure 1. User experience (UX) quantification model with value structure.

In other words, core and associated values should be identified to examine UX concept, according to the UX quantification framework mentioned above. The whole process of value structuring in this study is shown in Figure 2. First of all, a case study was carried out to assess participants' responses. Then, core and associated values were identified among significant keywords, which were extracted from user descriptions. Through applying network analyses on core values, information on associated values such as node strengths could help researchers formulate the value structure, which was verified with a post hoc analysis. 


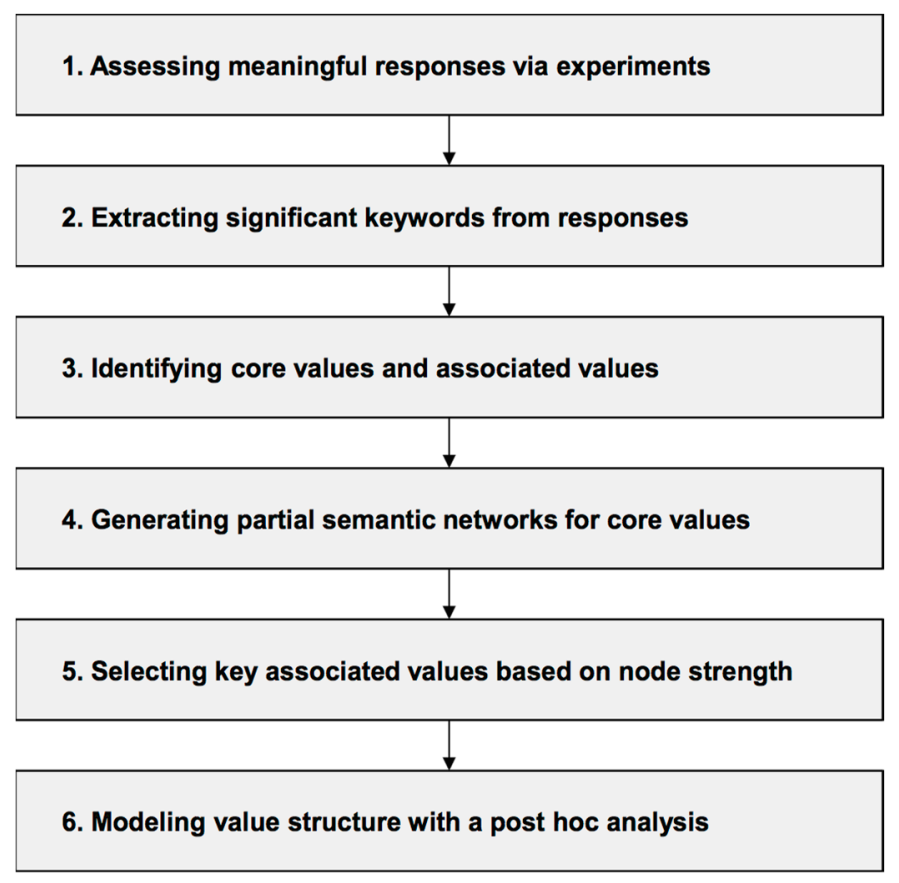

Figure 2. Value structuring process in this study.

\section{Case Study}

\subsection{Experimental Design}

In order to verify the ability of network analysis to build a value structure, a case study was used to gather user descriptions. Tasks were prescribed in detail to gain refined data. Hence, a value structure developed from this study is specialized for this specific set-up. Note that a value structure can vary over apparatus or context.

\subsubsection{Apparatus}

As an experimental device, a smart 55 inch TV was used to generate UXs. The manufacturer of the TV was Samsung (UNF8000), and the TV was available in Korea. Considering that UX varies over time and a user's perceived UX depends on previous experiences, an experimental device for the case study should have comparatively unfamiliar functions in order not to be affected by previous experiences. What this study cared about to do research was controlling experience rather than using new devices. That is why the experimental focus of this study was gesture interaction with smart TV. Although smart TVs are fairly common nowadays, gesture interactions with smart TV are less familiar.

\subsubsection{Participants}

A total of ten participants carried out the experimental tasks: five males and five females ranging in age from 20 to 35 years. Participants were recruited by announcements posted in online consumer electronics communities and retail electronics departments. In order to consider the potential users of smart TV gesture interactions and control the previous experience of users, technology-friendly participants (i.e., those familiar with the concept of gesture interaction) in their 20s and 30s were recruited as a priority.

Participants' information related to TV use is as follows: Two of them owned a smart TV and the other eight owned common LCD or LED TVs. Five of them owned a Microsoft Kinect and used it more than once a month, while four of them had experience with gesture interaction devices such as Microsoft Kinect or Nintendo Wii. Six participants watched TV for 2-3 h per day, two watched 1-2 $\mathrm{h}$ 
per day, and the others watched less than one hour per day. Note that this gesture interaction they experienced was different from the experimental prototype.

\subsubsection{Tasks}

Experimental tasks were predefined to control users' interactions with the smart TV that might affect their experiences and perceptions. Participants were asked to go through a total of nine tasks: turn on gesture mode, control the volume, change the channel, continuously adjust the volume and channel, access a specific page (e.g., the Smart Hub page which allows users to navigate smart functions), start a web browser, search for a certain word on the web browser, play gesture-based game (e.g., the Angry Birds), and free use. All tasks were based on gesture interactions.

\subsubsection{Procedure}

From the perspective of participants, the in-depth interview consisted of three parts: online survey, in-depth interview while interacting with the smart TV, and debriefing. Using the online survey, participants' basic demographic information and TV usage were gathered. Next, during the in-depth interview, participants' verbal comments, in Korean, were collected while they controlled the smart TV with their gestures. In this step, participants performed the nine tasks and were asked to describe their opinions and thoughts on the interactions. These descriptions were used as raw data for the network analysis. Finally, debriefing consisted of questionnaire items that sought additional opinions on the overall experiment.

\subsection{Results}

\subsubsection{Data Preprocessing}

During the in-depth interview about smart TV gesture interaction, a total of 240 meaningful responses were gathered from the ten participants. First, each response was decomposed into primary morphemes. As the smallest unit of meaning in a language, the morphemes were regarded as latent values. The Kind Korean Morpheme Analyzer (KKMA) 2.0 was used to decompose the responses into morphemes. Considering that meaningless morphemes such as postposition particles are frequently observed in responses in the Korean language, morphemes consisting of nouns, adjectives, verbs, adverbs, and determiners were selected from all the morphemes. As a result, 333 major morphemes were collected from meaningful responses.

For instance, one participant stated that "because the TV's accuracy is low, additional behavior such as an exaggerated gesture or loud voice is needed." In this case, morphemes including accuracy, low, additional, behavior, exaggerated, gesture, loud, and voice were selected. Of course, among them, there are still morphemes like 'low' that do not have any meaning in a standalone state and are hence meaningless.

Much of this was done by hand in a semiautomated way. In English, Natural Language Toolkit (NLTK) package of Python and various topic modeling algorithms can make it easy to analyze, but because the Korean language is complex, this study used a different approach. This study extracted words based on KKMA mentioned above, which is similar to the NLTK package, and investigated all the words that could be collected, rather than automatically extracting only the important words at this stage. Consequently, a semiautomated method required effort was used because the core of this method is in the development of the procedure.

Second, significant keywords were derived from the major morphemes. As mentioned above, morphemes that have no meaning independently were screened out. Morphemes that are synonyms were merged. For instance, at this stage, terms like fun and pleasure are merged into fun. Note that keywords intermingled core or associated values (e.g., convenience and fun) and design variables (e.g., type of control and gesture shape). As a result, a total of 132 nonoverlapping significant keywords were selected. 
Finally, co-occurrence matrices were generated using the set of significant keywords. In order to transform information into a dataset that could be analyzed with network analysis, a co-occurrence matrix was used. Each row indicates one response and includes several keywords described in the response. A response may have more than one keyword, while responses that do not have any keywords are filtered out. Here, keywords in the same row are defined as a co-occurrence. In other words, they may have relationships that be described as edges in the network structure. Note that associated values are defined as values that have a relationship with core values. As a result, a total of 251 co-occurrence rows composed the co-occurrence matrix.

\subsubsection{Network Analysis}

After applying network analysis to the co-occurrence matrix, the degree, betweenness, and eigenvector centralities of the nodes were extracted. Even though three centralities basically measure different properties of the network, high correlation coefficients among the centralities were recorded: 0.94 between degree and betweenness centralities, 0.87 between betweenness and eigenvector centralities, and 0.98 between degree and eigenvector centralities. Note that degree, betweenness, and eigenvector centrality respectively represent (1) the number of links incident on nodes, (2) the number of times a node acts as a bridge on the shortest path between two different nodes, and (3) relative scores of the number of important nodes to which a node is connected. As correlation coefficients turned out to be high, the lists of top-ranked nodes with respect to the three centralities were analogous (Table 1). Nodes such as convenience, gesture shape, fun, type of control, exactness, fatigue, desire to use, independent, range of motion, and repetitive ranked high among degree centralities.

Table 1. Centrality of representative nodes and their types.

\begin{tabular}{ccccc}
\hline Node & Degree * & Betweenness * & Eigenvector * & Node Type \\
\hline Convenience & $53(1)$ & $1262.8(1)$ & $0.295(1)$ & Value dimension ** \\
Gesture shape & $48(2)$ & $1192(3)$ & $0.252(2)$ & Design variable \\
Fun & $47(3)$ & $1196(2)$ & $0.23(4)$ & Value dimension ** \\
Type of control & $40(4)$ & $672.4(5)$ & $0.239(3)$ & Design variable \\
Exactness & $39(5)$ & $726.6(4)$ & $0.222(5)$ & Design variable \\
Fatigue & $34(6)$ & $518.6(7)$ & $0.222(5)$ & Value dimension ** \\
Desire to use & $33(7)$ & $612.9(6)$ & $0.202(8)$ & Value dimension \\
Independent & $29(8)$ & $412.7(8)$ & $0.205(7)$ & Value dimension \\
Range of motion & $27(9)$ & $317.9(11)$ & $0.166(10)$ & Design variable \\
Repetitive & $26(10)$ & $321.2(10)$ & $0.18(9)$ & Design variable \\
Novel & $26(10)$ & $371(9)$ & $0.161(12)$ & Value dimension \\
Learnability & $26(10)$ & $218.1(16)$ & $0.159(13)$ & Value dimension \\
Easy & $23(13)$ & $205.3(18)$ & $0.163(11)$ & Value dimension \\
Various & $21(14)$ & $232.5(15)$ & $0.125(17)$ & Design variable \\
Reaction time & $20(15)$ & $183.1(19)$ & $0.135(14)$ & Design variable \\
Quality of life & $20(15)$ & $271.6(12)$ & $0.111(20)$ & Value dimension \\
Familiar & $19(17)$ & $148.8(21)$ & $0.131(16)$ & Value dimension \\
Compatibility & $19(17)$ & $260(14)$ & $0.114(19)$ & Value dimension \\
Comfortable & $18(19)$ & $123.5(24)$ & $0.133(15)$ & Value dimension \\
Suspicious & $17(20)$ & $214.9(17)$ & $0.099(23)$ & Value dimension \\
Irritation & $17(20)$ & $170(20)$ & $0.094(27)$ & Value dimension \\
Error & $15(22)$ & $76.7(29)$ & $0.12(18)$ & Design variable \\
Stressful & $15(22)$ & $141.1(23)$ & $0.107(21)$ & Value dimension \\
Daily & $15(22)$ & $97.2(25)$ & $0.099(23)$ & Value dimension \\
Personalize & $15(22)$ & $66.5(31)$ & $0.091(30)$ & Value dimension \\
\hline The & & & \\
\end{tabular}

* The numbers in parentheses represent the ranks in the corresponding centrality. ${ }^{* *}$ Core value dimensions.

Each node was classified into two types: value dimensions and design variables. First, two criteria were used to identify a node as a design variable: (1) the node was a technical property of a product or service and (2) the node depends on the development of technology. For instance, because gesture 
shape and type of control are direct properties of gesture interaction, they were classified as design variables. Furthermore, exactness and reaction time are technical factors and hence also classified as design variables.

Second, among value dimensions, a certain value dimension was regarded as a core value dimension if it turned out to be important. In this study, if the degree centrality of a node was more than 33 (i.e., the node connected to more than $25 \%$ of all nodes in the network), then the node was designated a core value dimension. The criterion to divide value dimensions into core and associated values are expected to be further explored in future studies; judging whether a certain value is more important than another has no correct answer. The full semantic network is shown in Figure 3, along with the partial semantic network, which only shows nodes that have degree centralities of at least 15 and where eigenvector centralities are indicated by the size of each node. Note that this study used Ucinet and Gephi for applying network analysis.

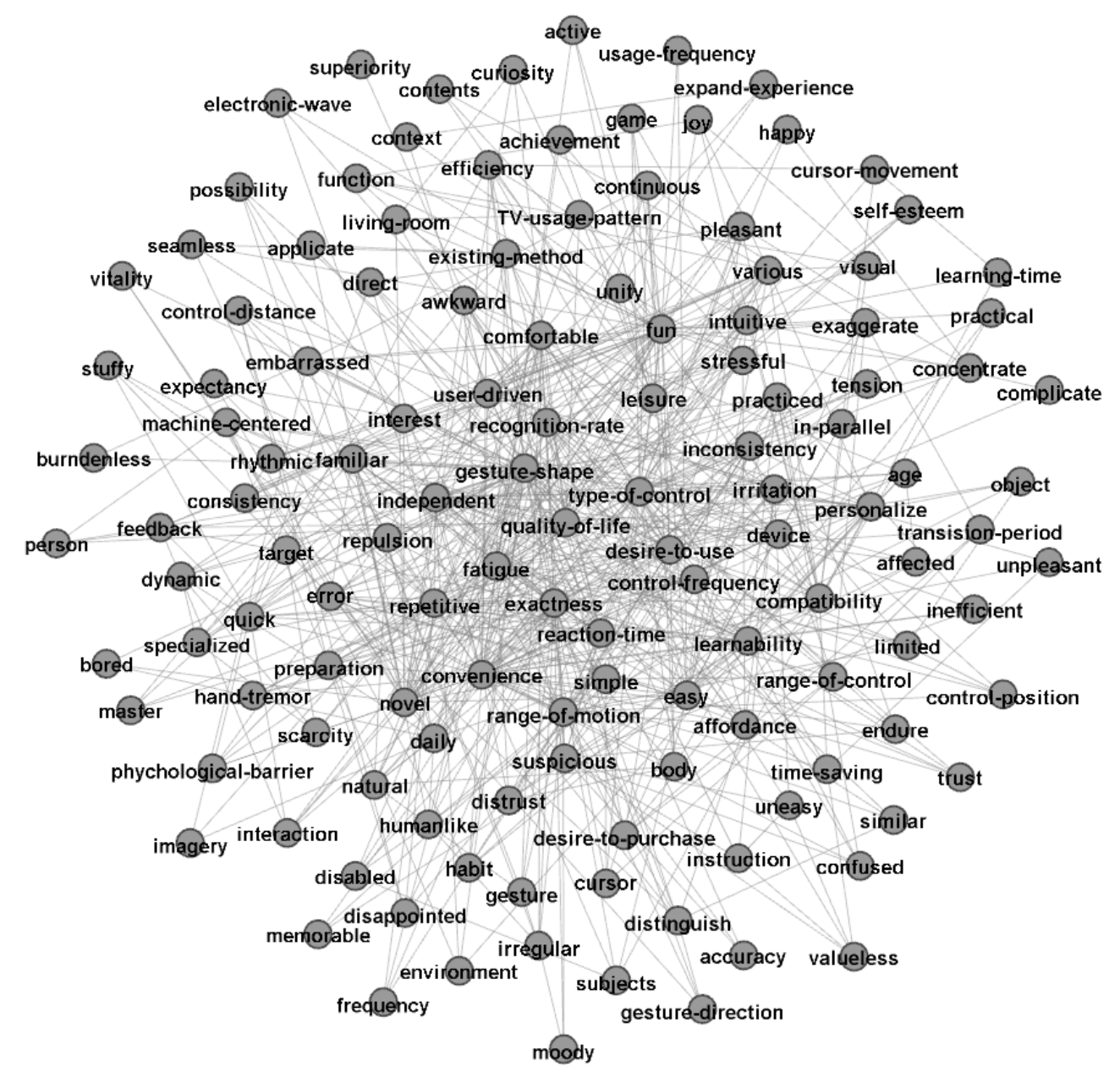

(a)

Figure 3. Cont. 


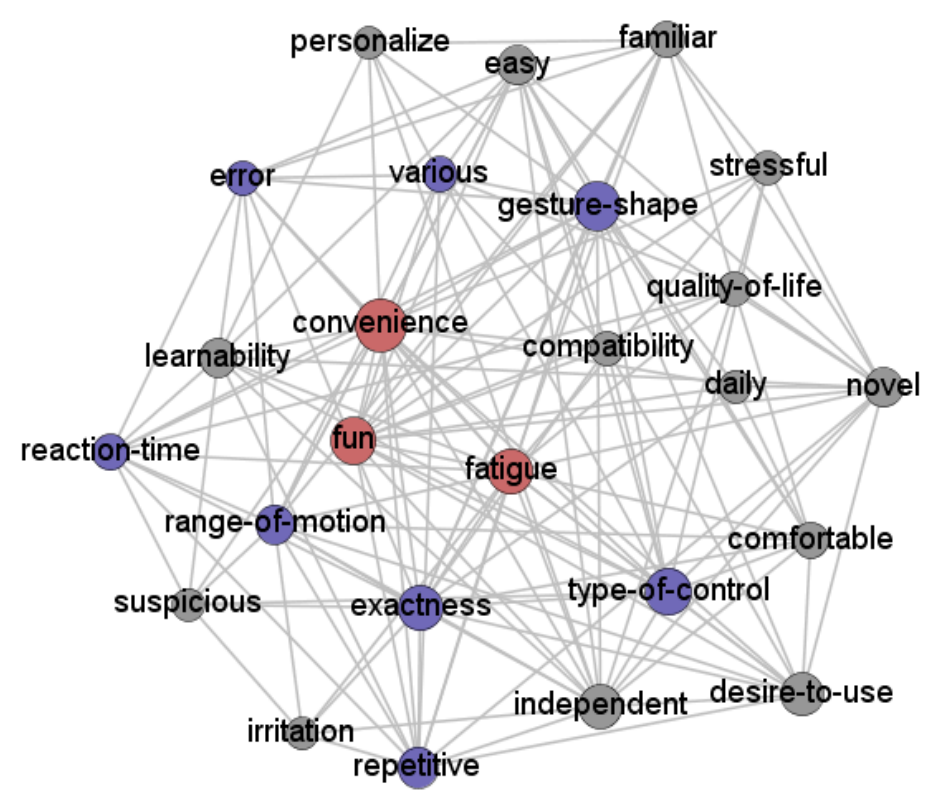

(b)

Figure 3. Semantic networks of smart TV gesture interactions. (a) Co-occurrence network of the 132 significant keywords. (b) Semantic network of representative nodes (red and blue nodes indicate core values and design variables, respectively).

Network Analysis of Convenience

As convenience was selected as a core value dimension, a co-occurrence matrix consisting of the values associated with convenience was constructed. The matrix includes 56 co-occurrence rows. Using network analysis, node-level centralities were then quantified again (Table 2). As a result, nodes such as type of control, fatigue, gesture shape, practiced, desire to use, learnability, easy, fun, age, repetitive, and range of motion turned out to be important in terms of degree centrality. While type of control, fatigue, and gesture shape were also regarded as important in the overall network analysis, nodes such as practiced were regarded as relatively more important in the convenience analysis. The semantic network belonging to convenience is shown in Figure 4, where nodes with degree centralities of at least eight are shown and eigenvector centralities are indicated as node size.

Table 2. Centrality of representative nodes associated with convenience.

\begin{tabular}{ccccc}
\hline Node & Degree * & Betweenness * $^{*}$ & Eigenvector * & Node Type \\
\hline Type of control & $27(1)$ & $120.7(1)$ & $0.301(1)$ & Design variable \\
Fatigue & $17(2)$ & $37.8(2)$ & $0.203(2)$ & Value dimension ** \\
Gesture shape & $15(3)$ & $36.1(3)$ & $0.137(11)$ & Design variable \\
Practiced & $13(4)$ & $16(4)$ & $0.176(3)$ & Value dimension \\
Desire to use & $12(5)$ & $13.8(5)$ & $0.164(4)$ & Value dimension \\
Learnability & $11(6)$ & $6(13)$ & $0.164(4)$ & Value dimension \\
Easy & $11(6)$ & $10.4(7)$ & $0.159(6)$ & Value dimension \\
Fun & $11(6)$ & $6.9(10)$ & $0.153(7)$ & Value dimension ** \\
Age & $10(9)$ & $7.3(9)$ & $0.149(8)$ & Value dimension \\
Repetitive & $10(9)$ & $7.4(8)$ & $0.122(16)$ & Design variable \\
Range of motion & $10(9)$ & $10.5(6)$ & $0.12(17)$ & Design variable \\
Doubtful & $9(12)$ & $1.9(15)$ & $0.142(10)$ & Value dimension \\
Distrust & $8(13)$ & $1.9(16)$ & $0.143(9)$ & Value dimension \\
Independent & $8(13)$ & $6.3(12)$ & $0.104(21)$ & Value dimension \\
Quick & $8(13)$ & $6.3(11)$ & $0.101(24)$ & Value dimension \\
\hline
\end{tabular}

* The numbers in parentheses represent ranks in the corresponding centrality. ${ }^{* *}$ Core value dimensions. 


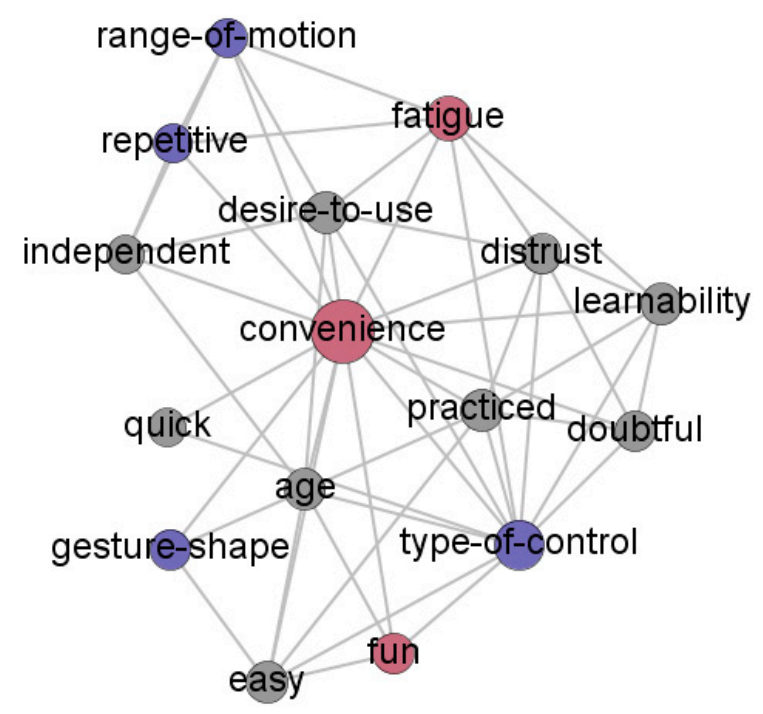

Figure 4. Partial semantic network of convenience (red and blue nodes indicate core values and design variables, respectively).

Network Analysis of Fun

As fun was selected to be the second core value dimension, a co-occurrence matrix that included the associated values associating with fun was constructed. A total of 59 co-occurrence rows composed the matrix. Just as for the network analysis of convenience, node-level centralities relating to fun were extracted (Table 3). As a result, nodes such as type of control, gesture shape, easy, various, convenience, good feeling, controllability, and age turned out to be important in terms of the degree centrality. Nodes such as good feeling and controllability turned out to be more important in the fun analysis than the overall network analysis. The semantic network belonging to fun is shown in Figure 5, where nodes with degree centralities of at least seven are shown and eigenvector centralities are indicated by node size.

Table 3. Centrality of representative nodes associated with fun.

\begin{tabular}{ccccc}
\hline Node & Degree * & Betweenness * & Eigenvector * & Node Type \\
\hline Type of control & $25(1)$ & $107.1(1)$ & $0.281(1)$ & Design variable \\
Gesture shape & $16(2)$ & $42.5(2)$ & $0.151(7)$ & Design variable \\
Easy & $14(3)$ & $17.2(4)$ & $0.197(2)$ & Value dimension \\
Various & $14(3)$ & $30(3)$ & $0.142(8)$ & Design variable \\
Convenience & $13(5)$ & $16.9(5)$ & $0.187(3)$ & Value dimension ** \\
Good feeling & $10(6)$ & $1.9(14)$ & $0.17(4)$ & Value dimension \\
Controllability & $10(6)$ & $1.9(14)$ & $0.17(4)$ & Design variable \\
Age & $10(6)$ & $1.9(14)$ & $0.17(4)$ & Value dimension \\
New & $9(9)$ & $7.8(8)$ & $0.124(11)$ & Value dimension \\
Leisure & $9(9)$ & $10.5(6)$ & $0.118(12)$ & Value dimension \\
Interest & $9(9)$ & $7.8(8)$ & $0.115(13)$ & Value dimension \\
Desire to use & $8(12)$ & $8(7)$ & $0.093(25)$ & Value dimension \\
Conventional & $7(13)$ & $0(30)$ & $0.142(8)$ & Value dimension \\
Novel & $7(13)$ & $3.8(11)$ & $0.113(14)$ & Value dimension \\
Quality of life & $7(13)$ & $4.5(10)$ & $0.087(28)$ & Value dimension \\
\hline
\end{tabular}

${ }^{*}$ The numbers in parentheses represent ranks in the corresponding centrality. ${ }^{* *}$ Core value dimensions. 


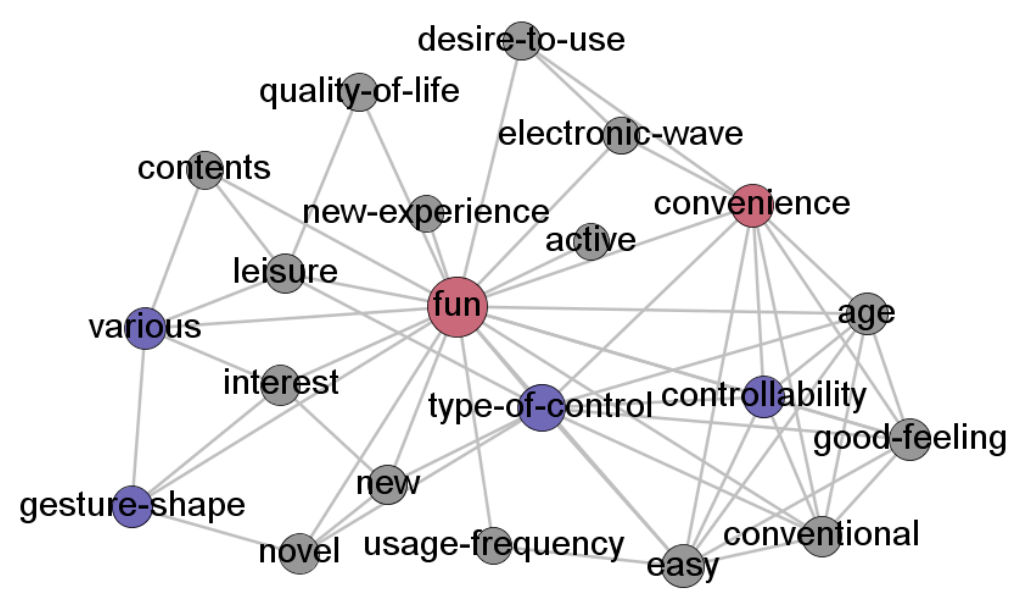

Figure 5. Partial semantic network of fun (red and blue nodes indicate core values and design variables, respectively).

\section{Network Analysis of Fatigue}

The third core value dimension fatigue was also separately analyzed with a co-occurrence matrix that included 52 co-occurrence rows. Node-level centralities were quantified (Table 4). As a result, nodes such as convenience, stressful, exactness, desire to use, irritation, range of motion, and subtle were regarded as important in terms of the degree centrality. Negatively described nodes such as stressful and irritation were unsurprisingly related to fatigue. The semantic network belonging to fatigue is shown in Figure 6, where only nodes with degree centralities of at least seven are shown and eigenvector centralities are indicated by node size.

Table 4. Centrality of representative nodes associated with fatigue.

\begin{tabular}{ccccc}
\hline Node & Degree * & Betweenness $^{*}$ & Eigenvector * & Node Type \\
\hline Convenience & $17(1)$ & $37.8(1)$ & $0.277(1)$ & Value dimension ** \\
Stressful & $15(2)$ & $32.8(2)$ & $0.205(3)$ & Value dimension \\
Exactness & $13(3)$ & $16.6(4)$ & $0.236(2)$ & Design variable \\
Desire to use & $13(3)$ & $21.6(3)$ & $0.191(5)$ & Value dimension \\
Irritation & $10(5)$ & $6.9(7)$ & $0.195(4)$ & Value dimension \\
Range of motion & $10(5)$ & $7.8(6)$ & $0.183(6)$ & Design variable \\
Subtle & $10(5)$ & $9.4(5)$ & $0.176(7)$ & Value dimension \\
Limited & $8(8)$ & $2.3(10)$ & $0.161(8)$ & Value dimension \\
Learnability & $8(8)$ & $5.7(8)$ & $0.122(18)$ & Value dimension \\
Intuitive & $7(10)$ & $2.8(9)$ & $0.157(9)$ & Value dimension \\
Hand tremor & $7(10)$ & $0.2(27)$ & $0.157(9)$ & Value dimension \\
Control frequency & $7(10)$ & $0.2(27)$ & $0.157(9)$ & Design variable \\
Repetitive & $7(10)$ & $0.2(27)$ & $0.157(9)$ & Design variable \\
Range of control & $7(10)$ & $0.6(16)$ & $0.143(13)$ & Design variable \\
\hline
\end{tabular}

\footnotetext{
${ }^{*}$ The numbers in parentheses represent ranks in the corresponding centrality. ${ }^{* *}$ Core value dimensions.
} 


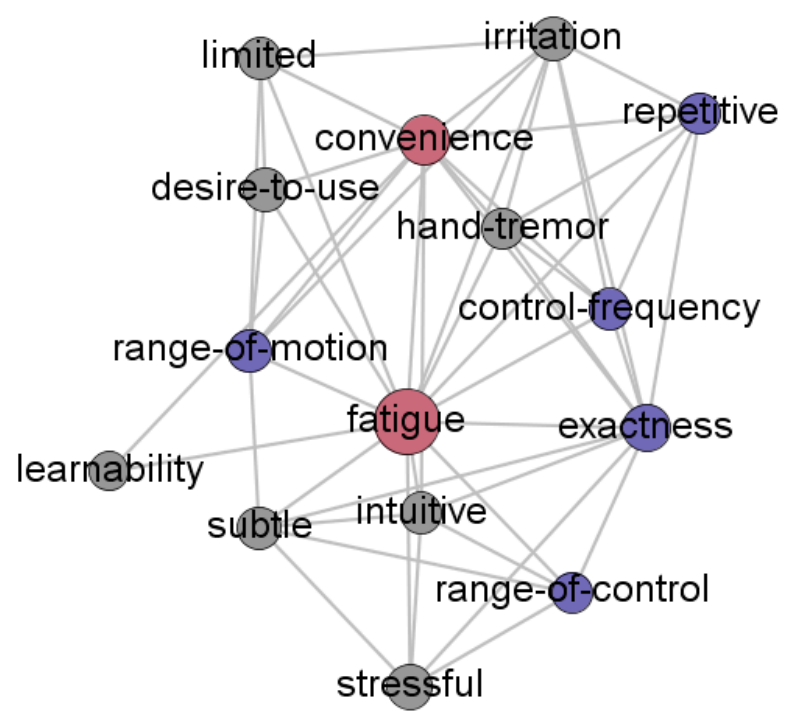

Figure 6. Partial semantic network of fatigue (red and blue nodes indicate core values and design variables, respectively).

\subsubsection{Formulation of Value Structure}

Based on the information conveyed by nodes and edges, several formulae can be drawn up. It is assumed that node strength is proportional to the number of repetitions. First, the formation of the overall UX was assumed to consist of three core value dimensions according to the research framework in this study. Second, the formation of each core value dimension was filtered by node strength. When applying the min-max normalization to node strengths, dimensions of more than 0.3 were selected.

As a result, several nodes were considered to affect core value dimensions such as convenience, fun, and fatigue. Nodes such as practiced, learnability, doubtful, tension, desire to use, and gesture shape were regarded to affect convenience; nodes such as tension, easy, gesture shape, and new were assumed to affect fun; nodes such as limited, range of motion, stressful, desire to use, learnability, exactness, subtle, type of control, seamless, error, and independent were considered to affect fatigue.

In order to refine the value structure, a post hoc analysis with an experiment was carried out. Considering the case study had a limitation to probe into gestural interactions with only qualitative descriptions, a post hoc analysis aimed to assess quantitative measurements which would be used to verify the result of the case study.

Experimental tasks were repeated with the same experimental device without an in-depth interview, but each participant was required to evaluate gesture interactions psychophysically. A total of 21 influential dimensions (Table 5) were rated with the 0-10 scale, utilizing the psychophysical measurements used in previous studies [34-36]. Note that design variables such as type of control were also included the questionnaire items for building models.

The simple linear regression method was applied to build value structure models, based on a total of twenty participants' evaluation data. The variables consisted of the core linkage concept in the network analysis output. When applying the method, a dimension whose variation inflation factor (VIF) was higher than 10 was removed from the value structure models to minimize multi-collinearity [37]. As a result, totally four models were built, whose R2 and the maximum VIF were 0.70 and 4.53 on the average (Table 6). 
Table 5. Influential value dimensions and their definitions.

\begin{tabular}{|c|c|}
\hline Dimension & Definition \\
\hline Convenience & The system is convenient for me \\
\hline Practiced & There are many features that I practiced in using \\
\hline Learnability & I can learn to use this system very quickly \\
\hline Doubtful & I am doubtful about using the system \\
\hline Tension & I feel quite tense when I use this system \\
\hline Desire to use & I have a desire to use the system \\
\hline Gesture shape & Gesture shapes used in this system are intuitive or novel \\
\hline Fun & The interface of this system is fun to use \\
\hline Easy & I think the system is easy to use \\
\hline New & I am attracted to novel features \\
\hline Fatigue & I feel physical fatigue when I use this system \\
\hline Limited & The extent of use is limited \\
\hline Range of motion & The system covers the wide range of motion \\
\hline Stressful & Using this system is stressful \\
\hline Exactness & The system can recognize user intention exactly \\
\hline Subtle & I can convey subtle and delicate expressions to the system \\
\hline Type of control & Type of control itself seems to be easy and convenient \\
\hline Seamless & An interaction with the system works in a seamless manner \\
\hline Error & Many errors occur when I use the system \\
\hline Independent & The system does not depend on a specific device or service \\
\hline Overall & Overall, I am satisfied with this system \\
\hline
\end{tabular}

Table 6. Model equations extracted from node strengths.

\begin{tabular}{cccc}
\hline Dependent Variable & $\mathbf{R}^{\mathbf{2}}$ & Max. VIF & Model Equation \\
\hline Overall UX & 0.78 & 2.362 & $4.77+0.505 \times$ Convenience $+0.346 \times$ Fun $-0.68 \times$ Fatigue \\
\hline Convenience & 0.71 & 5.468 & $\begin{array}{c}2.765-0.046 \times \text { Practiced }+0.083 \times \text { Learnability }+0.091 \times \\
\text { Doubtful }-0.446 \times \begin{array}{c}\text { Tension }+0.623 \times \text { Desire to use }-0.02 \times \\
\text { Gesture shape }\end{array}\end{array}$ \\
\hline Fun & 0.69 & 3.100 & $-0.385+0.116 \times \begin{array}{c}\text { Tension }+0.566 \times \text { Easy }+0.768 \times \text { Gesture } \\
\text { shape }-0.355 \times \text { New }\end{array}$ \\
\hline Fatigue & 0.60 & 7.192 & $\begin{array}{l}7.906-0.125 \times \text { Limited }-0.189 \times \text { Range of motion }-0.021 \\
\times \text { Stressful }+0.062 \times \text { Desire to use }-0.025 \times \text { Learnability }+ \\
0.08 \times \text { Exactness }+0.111 \times \text { Subtle }-0.317 \times \text { Type of control } \\
+0.103 \times \text { Seamless }+0.034 \times \text { Error }\end{array}$ \\
\hline
\end{tabular}

* Max. indicate maximum.

\subsection{Discussion}

\subsubsection{Compatibility between Value Structure and Network Analysis}

Although network analysis has been applied to various fields, the results of network analysis must be checked to determine whether they agree with value structuring or not. In the field of social network analysis, nodes are main agents that can have relationships with other agents, including humans and animals. Of course, in some network analyses, abstract concepts such as web pages have been used as nodes [26]. Regardless, nodes and edges should be carefully designated.

In this study, constructs were defined as nodes, while associations among the constructs were defined as edges. Constructs include heterogeneous elements such as values or design components, while associations among the constructs strictly represent implicit relationships among the elements. Consequently, associations among the constructs form the groundwork for the application of network analysis to value structuring. Note that, as a widely-used methodology in the field of psychology, 
the implicit-association test (IAT) assumes that associations among mental representations of objects have various strengths [38], and forms a similar structure to the one proposed in this study.

In spite of their compatibility, there are two limitations of value structures built using network analysis. First, the value structure reflects the current core and associated values. Even if a UX and its value dimensions vary over time, an experiment can only reflect the current temporal state. Second, latent or unconscious values are difficult to detect through an experiment. Latent or unconscious values cannot help appearing with missing or weak node strengths. Alternatively, participants may not even mention these values during an experiment. Compensation for latent or unconscious values is expected to be addressed in further study.

\subsubsection{Properties of Value}

The value itself is an ambiguous concept. Although whether a value was identified to be a core value or an associated value was determined by the degree of influence in this study, whether a certain term deserved to be called a value was not strictly verified. The reason for this is that this study assumes an individual can pursue any state or mode that might be expressed using any term. Note that values can be defined as desirable states of existence or modes of behavior [13].

Consequently, different types of values were included in the value structure developed in this study. For example, a value such as happy may be regarded as a desirable end-state of existence, while a value such as learnability may be regarded as an instrumental concept and have high relevance to a certain product or service. Values or needs are widely known to have various levels and perspectives. [39] suggested five phases an individual could encounter: physiological, safety, love, esteem, and self-actualization.

In addition, a negative term such as fatigue was allowed to be included in the value structure in this study. When a user mentions a negative term, it can be generally assumed that the user wants to avoid the situation accompanying that term. Conversely, if an appropriate term that expresses a user's mental state is discovered, the appropriate term may be the alternative of a positive value. For an instant, freshness or relaxation could replace the term fatigue. However, considering the automatic development of the value structure using network analysis, negative terms were, in practice, included in the value structure.

\subsubsection{Limitation of UX Quantification}

Basically, quantifying UX causes loss of information. In this case, user descriptions were transformed into numerical and relational information. With respect to the amount of information, a certain amount of information is unavoidably left out during transformation. As experiences of users are diverse, most UX research has the same limitation.

Of course, even if the amount of information conveyed by a network analysis is small, that information seems to be highly relevant to decision making. Moreover, a value-structured method might be expected to lose less information than traditional usability or UX quantification methods in terms of enhancing the diversity of assessment dimensions.

\subsubsection{Verification of Value Structure}

One of the limitations of this study itself was the small number of participants to derive data for network analysis. It is possible that this sample had a biased experience with ten participants. Of course, statistical analyses which usually have conservative assumptions were not applied, and it is not a critical problem. Nonetheless, additional experiments were carried out to supplement this. This study tried to evaluate the value structure and the reliability of the main experiment through further experiments.

Although the performance of the value structure models revealed that they would be reliable, some considerations seem to be required in this study. First, value dimensions, which were used in the post hoc analysis, should be assumed to be defined correctly, even if an essentialistic interpretation of 
any definition on a certain construct could distort the meaning [16]. Second, the coefficients of models should be interpreted carefully and empirically. For example, a value dimension desire to use turned out to be positively related to fatigue. For clarification over a certain construct, additional work using tools such as structural equation modeling would be needed.

Of course, there could be various methods to formulate a value structure. For example, noncompensatory equations such as conjunctive and disjunctive models [40] or nonlinear equations such as the S-shaped value model [41] should be verified. Validation of the equations extracted from the network structure will be carried out in future work. The focus of this study is to confirm the possibility of building hierarchical value structures.

\subsubsection{Application of the Framework}

The framework, which is the result of this research, can be applied to the analysis of various products or services, although this study is limited to gestural interaction. First, it can be used as a reference for researching and making decisions in the planning stage before developing a specific product or service. Decision-making can save you a whole cost if it goes as early as possible. This framework is based on the interview data of potential users so that the area of products or services can be all kinds that can be produced by enterprises. Second, the framework can be applied to not only commercial products but also new technologies, organizations, and abstract concepts that do not exist. It is expected that this will be helpful for academic research and research activities of government and corporations.

\section{Conclusions}

This study proposed a semantic map, a so-called value structure that is fit to a product or service usage so that it may be used as an evaluation framework. Values may vary over contexts and target products or services. Hence, a value structure for a certain product or service may vary. To confirm the possibilities of developing a value structure using network analysis, this study conducted a case study of smart TV gesture interactions. Network analysis was then used to extract representative value dimensions from participant response during and after gesture interactions.

In terms of gesture interaction on smart TVs, convenience, fun, and fatigue turned out to be core value dimensions among associated value dimensions such as a desire to use, independent, novel, learnability, easy, quality of life, familiar, and compatibility. Some nodes such as gesture shape, type of control, and exactness were designated as design variables, which were supposed to be properties of smart TV gesture interactions or to depend on system performance. In addition, value structure models, based on node strengths, were formulated and confirmed with a post hoc analysis.

One of the major contributions of network analysis is to transfer qualitative descriptions to quantitative data. Users' perceptions or opinions generally consist of narrative descriptions. Once evaluation dimensions such as the luxuriousness of a certain product are given, users may rate the product on a certain numerical scale. However, manipulated numbers risk distorting UX in reality. If a user's descriptions toward a specific product or service can be quantitatively analyzed, the loss of information can be decreased.

Although this study suggested a methodology for building a value structure for a specific product or service, it could have limitations, and future work remains to be done. First, probing for latent or unconscious values, some of which may turn out to be important, should be explored to enhance compatibility between value structure and network analysis. Second, applying network analysis to other products or services should be done to validate the reliability of the framework. Consequently, the value structuring method suggested in this study is expected to be used by designers to examine alternative analyses of various product or service families. 
Author Contributions: Conceptualization and methodology, J.H.L.; formal analysis, Y.L.R.; writing and editing, J.H.P.

Funding: This work was supported by the Incheon National University Research Grant in 2018 (Grant No.: 2018-0242).

Conflicts of Interest: The authors declare no conflict of interest.

\section{References}

1. International Organization for Standardization. Ergonomic Requirements for Office Work with Visual Display Terminals (VDTs). Part 11: Guidance on Usability; ISO Standard 9241-11:1998; International Organization for Standardization: Geneva, Switzerland, 1998.

2. Han, S.H.; Yun, M.H.; Kwahk, J.; Hong, S.W. Usability of consumer electronic products. Int. J. Ind. Ergon. 2001, 28, 143-151. [CrossRef]

3. Kwahk, J.; Han, S.H. A methodology for evaluating the usability of audiovisual consumer electronic products. Appl. Ergon. 2005, 33, 419-431. [CrossRef]

4. International Organization for Standardization. Ergonomics of Human System Interaction. Part 210: Human-Centered Design for Interactive Systems; ISO Standard 9241-210:2009; International Organization for Standardization: Geneva, Switzerland, 2009.

5. Alben, L. Quality of experience: Defining the criteria for effective interaction design. Interactions 1996, 3 , 11-15. [CrossRef]

6. Kuniavsky, M. User experience and HCI. In The Human-Computer Interaction Handbook: Fundamentals, Evolving Technologies, and Emerging Applications, 2nd ed.; Sears, A., Jacko, J.A., Eds.; Lawrence Erlbaum Associates: New York, NY, USA, 2007.

7. McNamara, N.; Kirakowski,J. Functionality, usability, and user experience: Three areas of concern. Interactions 2006, 13, 26-28. [CrossRef]

8. Park, J.; Han, S.H.; Kim, H.K.; Cho, Y.; Park, W. Developing elements of user experience for mobile phones and services: Survey, interview, and observation approaches. Hum. Factors Ergon. Manuf. Serv. Ind. 2013, 23, 279-293. [CrossRef]

9. Park, J.; Han, S.H.; Kim, H.K.; Moon, H.; Park, J. Developing and verifying a questionnaire for evaluating user value of a mobile device. Hum. Factors Ergon. Manuf. Serv. Ind. 2015, 25, 724-739. [CrossRef]

10. Hassenzahl, M.; Tractinsky, N. User experience-A research agenda. Behav. Inf. Technol. 2006, 25, 91-97. [CrossRef]

11. Karapanos, E.; Zimmerman, J.; Forlizzi, J.; Martens, J.B. User experience over time: An initial framework. In Proceedings of the 27th International Conference on Human Factors in Computing Systems (CHI 2009), Bostom, MA, USA, 4-9 April 2009; pp. 729-738.

12. Kim, H.K.; Han, S.H.; Park, J.; Park, W. How user experience changes over time: A case study of social network services. Hum. Factors Ergon. Manuf. Serv. Ind. 2015, 25, 659-673. [CrossRef]

13. Rokeach, M. Beliefs, Attitudes and Values: A Theory of Organization and Change; Jossey-Bass Inc.: San Francisco, CA, USA, 1968.

14. Gutman, J. A means-end chain model based on consumer categorization processes. J. Mark. 1982, 46, 60-72. [CrossRef]

15. Park, J.; Han, S.H. A value sampling method for evaluating user value: A case study of a smartphone. Int. J. Mo. Com. 2018, 16, 440-458. [CrossRef]

16. Park, J.; Han, S.H. Defining user value: A case study of a smartphone. Int. J. Ind. Ergon. 2013, 43, $274-282$. [CrossRef]

17. Swidler, A. Culture in action: Symbols and strategies. Am. Sociol. Rev. 1986, 51, 273-286. [CrossRef]

18. Nielsen, J.; Molich, R. Heuristic evaluation of user interfaces. In Proceedings of the ACM CHI 90 Human Factors in Computing Systems Conference (CHI 90), Seattle, WA, USA, 1-5 April 1990; pp. 249-256.

19. Deary, I.J.; Der, G.; Ford, G. Reaction times and intelligence differences: A population-based cohort study. Intelligence 2001, 29, 389-399. [CrossRef]

20. Lim, J.H.; Ryu, T.; Kim, Y. Effects of visual stimulus on response behavior of control-on-display interface. Int. J. Hum. Comput. Interact. 2014, 30, 291-302. [CrossRef]

21. Wilson, E.O. Sociobiology: The New Synthesis; Harvard University Press: Cambridge, MA, USA, 1975. 
22. Bezanson, M.; Garber, P.A.; Murphy, J.T.; Premo, L.S. Patterns of subgrouping and spatial affiliation in a community of mantled howling monkeys (Alouatta palliata). Am. J. Primatol. 2008, 70, 282-293. [CrossRef] [PubMed]

23. Croft, D.P.; James, R.; Thomas, P.O.R.; Hathaway, C.; Mawdsley, D.; Laland, K.N.; Krause, J. Social structure and co-operative interactions in a wild population of guppies (Poecilia reticulata). Behav. Ecol. Sociobiol. 2005, 59, 644-650. [CrossRef]

24. Lusseau, D.; Wilson, B.; Hammond, P.S.; Grellier, K.; Durban, J.W.; Parsons, K.M.; Barton, T.R.; Thompson, P.M. Quantifying the influence of sociality on population structure in bottlenose dolphins. J. Anim. Ecol. 2006, 75, 14-24. [CrossRef] [PubMed]

25. Newman, M.E.J. The structure and function of complex networks. SIAM Rev. 2003, 45, 167-256. [CrossRef]

26. Kleinberg, J.M. Authoritative sources in a hyperlinked environment. J. ACM 1999, 46, 604-632. [CrossRef]

27. Shannon, P.; Markiel, A.; Ozier, O.; Baliga, N.S.; Wang, J.T.; Ramage, D.; Amin, N.; Schwikowski, B.; Ideker, T. Cytoscape: A software environment for integrated models of biomolecular interaction networks. Genome Res. 2003, 13, 2498-2504. [CrossRef]

28. Anderson, J.R.; Bower, G.H. Human Associative Memory; Winston: Washington, DC, USA, 1973.

29. Collins, A.M.; Loftus, E.F. A spreading-activation theory of semantic processing. Psychol. Rev. 1975, 82, 407-428. [CrossRef]

30. Pang, B.; Lee, L. Opinion mining and sentiment analysis. Found. Trends Inf. Retr. 2008, 2, 1-135. [CrossRef]

31. Schindler, R.M.; Bickart, B. Published word of mouth: Referable, consumer-generated information on the internet. In Online Consumer Psychology: Understanding and Influencing Consumer Behavior in the Virtual World; Haugtvedt, C.P., Machleit, K.A., Yalch, R.F., Eds.; Lawrence Erlbaum Associates: Hillsdale, NJ, USA, 2005.

32. Hoser, B.; Hotho, A.; Jaschke, R.; Schmitz, C.; Stumme, G. Semantic network analysis of ontologies. In Proceedings of the 3rd European Semantic Web Conference (ESWC 2006), Budva, Montenegro, 11-14 June 2006.

33. Leydesdorff, L.; Welbers, K. The semantic mapping of words and co-words in contexts. J. Informetr. 2011, 5, 469-475. [CrossRef]

34. Brooke, J. SUS: A quick and dirty usability scale. In Usability Evaluation in Industry; Jordan, P.W., Thomas, B., Weerdmeester, B.A., McClelland, A.L., Eds.; Taylor and Francis: London, UK, 1986.

35. Kirakowski, J.; Corbett, M. SUMI: The software usability measurement inventory. Br. J. Educ. Technol. 2006, 24, 210-212. [CrossRef]

36. Park, J.; Han, S.H.; Kim, H.K.; Oh, S.; Moon, H. Modeling user experience: A case study on a mobile device. Int. J. Ind. Ergon. 2013, 43, 187-196. [CrossRef]

37. Kutner, M.H.; Christopher, J.N.; Neter, J.; William, L. Applied Linear Statistical Models, 5th ed.; McGraw-Hill: New York, NY, USA, 2005.

38. Greenwald, A.G.; McGhee, D.E.; Schwartz, J.K.L. Measuring individual differences in implicit cognition: The implicit association test. J. Personal. Soc. Psychol. 1998, 74, 1464-1480. [CrossRef]

39. Maslow, A.H. A theory of human motivation. Psychol. Rev. 1943, 50, 370-396. [CrossRef]

40. Coombs, C.H. A Theory of Data; Wiley: New York, NY, USA, 1964.

41. Kahneman, D.; Tversky, A. Prospect theory: An analysis of decisions under risk. Econometrica 1979, 47, 313-327. [CrossRef]

(C) 2019 by the authors. Licensee MDPI, Basel, Switzerland. This article is an open access article distributed under the terms and conditions of the Creative Commons Attribution (CC BY) license (http://creativecommons.org/licenses/by/4.0/). 